\title{
Multiple Object Tracking Using Particle Filters
}

\author{
M. Jaward, L. Mihaylova, N. Canagarajah and D. Bull \\ Department of Electrical and Electronic Engineering, University of Bristol \\ Woodland Road, Bristol, BS8 1UB, UK \\ m.h.jaward@bristol.ac.uk, mila.mihaylova@ieee.org
}

\begin{abstract}
The particle filtering technique with multiple cues such as colour, texture and edges as observation features is a powerful technique for tracking deformable objects in image sequences with complex backgrounds. In this paper, our recent work [1] on single object tracking using particle filters is extended to multiple objects. In the proposed scheme, track initialisation is embedded in the particle filter without relying on an external object detection scheme. The proposed scheme avoids the use of hybrid state estimation for the estimation of number of active objects and its associated state vectors as proposed in [2]. The number of active objects and track management are handled by means of probabilities of the number of active objects in a given frame. These probabilities are shown to be easily estimated by the Monte Carlo data association algorithm used in our algorithm.
\end{abstract}

The proposed particle filter (PF) embeds a data association technique based on the joint probabilistic data association (JPDA) which handles the uncertainty of the measurement origin. The algorithm is able to cope with partial occlusions and to recover the tracks after temporary loss. The probabilities calculated for data associations take part in the calculation of probabilities of the number of objects. We evaluate the performance of the proposed filter on various real-world video sequences with appearing and disappearing targets.

\section{TABLE OF CONTENTS}

\section{INTRODUCTION}

2 Problem Formulation

3 Multiple target tracking

4 Joint Probability Data Association

5 EXPERIMENTAL RESULTS

6 Discussion

7 CONClusions

\section{INTRODUCTION}

Tracking a group of targets in a video sequence is a common problem in many video surveillance applications. Video surveillance addresses real-time observation of targets such as humans or vehicles in some environment, leading to a description of the objects' activities with the environment or among them. It has been used for security monitoring [3], [4],

0-7803-9546-8/06/ \$20.00/ @ 2006 IEEE

IEEEAC paper \# 1280 as well as for traffic flow measuring [5], accident detection on highways, and routine maintenance in nuclear facilities.

This paper addresses the problem of tracking multiple targets in video sequences. The objective is to detect and track targets which can undergo non-rigid deformations, rotations or partial occlusions. Tracking is based on multiple independent particle filters and the Probabilistic Data Association (PDA) algorithm which afford to handle the uncertainty due to the measurement origin. The data association algorithm also helps to recover from partial occlusions. The proposed algorithm can estimate the number of active targets in the video sequence and can accordingly increase the number of tracking filter(s) or stop unwanted tracking filter(s).

In many of the previously developed tracking techniques, the first fundamental problem encountered is the object segmentation which extracts the areas (or objects) of interest from the scene. The extracted regions are used as measurements (observations) for tracking algorithms. These foreground segmentation techniques are typically computationally expensive because they operate over the whole image scene [6].

Algorithms which attempt to find the target of interest without using segmentation have been proposed for single target tracking based on cues such as colour, edges and textures [1]. Recently, the integrated detection and tracking using particles has been extended for multiple targets. Many of these schemes rely on hybrid sequential state estimation. In [2], the state vector denoting all the existing targets is augmented by a discrete random variable which represents the number of existing objects in a video sequence. The particle filter developed in [7] has multiple models for the object motion, and comprises an additional discrete state component, denoting which of the motion models is active. The Bayesian Multiple-Blob Tracker (BraMBLe) [8] presents a multipleperson tracking system based on statistical appearance models. The multiple blob tracking is managed by incorporating the number of objects present in the state vector and state vector is augmented as in [2] when a new object enters the scene.

The problem of multiple target tracking is more challenging than the single target tracking and several issues which do not exist for single target tracking, has to be resolved for the successful application of multiple target tracking algorithms. One issue is the management of multiple tracks caused by newly appearing targets and the disappearance of already existing targets. In the references mentioned before, this is 
handled by the hybrid state estimation framework for joint tracking of all existing targets (with an extended state vector). Joint tracking of targets avoids the possibility of several independent filters, but the identity of the individual targets might be lost, especially when targets are close to each other. A separate single tracking filter can be applied to multiple target tracking, only when all targets are fairly well spaced.

When the targets are closer and/or cross each other, it has been shown in aerospace applications [9], [10], [11], that data association techniques can be used to track without loosing the identity of each targets. However, the methods developed in [12] for radar data are not applicable to vision problems due to the absence of measurement equations in explicit form. In [12] the varying number of targets is estimated via clustering techniques and the data association is performed by the 2-D assignment algorithm (see e.g. the review from Chapter 2 of [13])

In this paper, a sequential Monte Carlo version of the data association scheme is presented for tracking multiple targets and the track management is handled by existence probabilities calculated from the data association stage. This proposed scheme is simple and does not demand high computational resources.

This paper is organised as follows. The model of the moving object is described in section 2. The particle filter (PF) JPDA algorithm is given in section 3. Section 4 yields the new implemented Joint PDA scheme. Section 5 contains the experimental results, followed by a discussion in section 6 . Finally, the conclusions are highlighted in section 7.

\section{PROBLEM Formulation}

The aim is to sequentially perform simultaneous detection and tracking of objects described by the same specified colour histogram $q^{*}$, in a video sequence $\boldsymbol{Z}_{k}=\left\{\boldsymbol{z}_{1}, \boldsymbol{z}_{2}, \ldots, \boldsymbol{z}_{k}\right\}$, where $\boldsymbol{z}_{k}$ denotes the image (vector of pixel values) at discrete time instant $k$. The state space approach requires to specify a motion model, i.e., the evolution of the state $p\left(\boldsymbol{x}_{k} \mid \boldsymbol{x}_{k-1}\right)$, and a measurement model, i.e., the link between state and current measurement $p\left(\boldsymbol{z}_{k} \mid \boldsymbol{x}_{k}\right)$. The next two subsections describe the model of the object motion and the measurement likelihood function.

\section{State vector and dynamic model}

The state vector at frame $k$ of a single object typically consists of kinematic and region (or shape) parameters. For simplicity we use the random walk model, with the following state vector $\boldsymbol{x}_{k}=\left[x_{k}, y_{k}\right]^{T}$, where $\left(x_{k}, y_{k}\right)$ denotes the center of the image region used for the colour histogram computation. Note that other variables can be added, such as width and height of image region, velocities and scale change rate, depending on the application. The state dynamics is typically described by a linear model:

$$
\boldsymbol{x}_{k}=\boldsymbol{F} \boldsymbol{x}_{k-1}+\boldsymbol{v}_{k-1}
$$

where $\boldsymbol{F}$ is the transition matrix ( $\boldsymbol{F}=\boldsymbol{I}$ for our random walk model) and $\boldsymbol{v}_{k-1}$ is the process noise, assumed to be white, zero-mean, Gaussian, with a covariance matrix $Q$.

\section{Colour measurement model}

The work described in this paper is based on colour measurement cue. Other measurements cues, e.g. texture, edges or motion and joint variations of any of them can be used as the measurement cue. The choice of the specific cue depends on many factors such as the resolution of the video, background/foreground environment and dynamic nature of the targets to be tracked. Following [14], [15], we do not use the entire image $\boldsymbol{z}_{k}$ as a measurement, but rather we extract from the image the colour histogram $q_{k}$, computed inside the image region that is specified by the state vector $\boldsymbol{x}_{k}$. The center is defined by $\left(x_{k}, y_{k}\right)$. Furthermore, we adopt the Gaussian density for the likelihood function of the measured colour histogram as follows:

$$
p\left(q_{k} \mid \boldsymbol{x}_{k}\right) \propto \mathcal{N}\left(D_{k} ; 0, \sigma^{2}\right)=\frac{1}{\sqrt{2 \pi} \sigma} \exp \left\{-\frac{D_{k}^{2}}{2 \sigma^{2}}\right\},
$$

where $D_{k}$ is the distance between the reference histogram $q^{*}$ of objects to be tracked and the histogram $q_{k}$ computed from the current frame $\boldsymbol{z}_{k}$ in the region specified by the state vector $\boldsymbol{x}_{k}$. The standard deviation $\sigma$ of the Gaussian density in (2) is a design parameter.

If the two histograms are calculated over $U$ bins, the distance $D_{k}$ between two histograms is derived in [14] from the Bhattacharya similarity coefficient and defined as:

$$
D_{k}^{2}=1-\sum_{u=0}^{U} \sqrt{q^{*}(u) q_{k}(u)}
$$

\section{Multiple tARGet TRACKing}

The multiple target tracking is based on a sequential Monte Carlo filter and a Monte Carlo data association is avoiding the ambiguities caused by the different measurement origin. In this section, we explain the conceptual ideas behind the particle filter.

\section{Sequential Monte Carlo}

Sequential Monte Carlo techniques also known as particle filtering and condensation algorithm and their applications in the specific context of visual tracking, have been described in length in the literature [16], [17], [18].

For tracking the object of interest, the posterior state distribution $p\left(\boldsymbol{x}_{k} \mid \boldsymbol{Z}_{k}\right)$, also known as filtering distribution has to be calculated at each time step. In Bayesian sequential estimation the filtering distribution can be computed according to the two step recursion: prediction step

$$
p\left(\boldsymbol{x}_{k} \mid \boldsymbol{Z}_{k-1}\right)=\int p\left(\boldsymbol{x}_{k} \mid \boldsymbol{x}_{k-1}\right) p\left(\boldsymbol{x}_{k-1} \mid \boldsymbol{Z}_{k-1}\right) d \boldsymbol{x}_{k-1}
$$

and 


\section{filtering step}

$$
p\left(\boldsymbol{x}_{k} \mid \boldsymbol{Z}_{k}\right) \propto p\left(\boldsymbol{z}_{k} \mid \boldsymbol{x}_{k}\right) p\left(\boldsymbol{x}_{k} \mid \boldsymbol{Z}_{k-1}\right),
$$

where the prediction step follows from marginalisation, and the new filtering distribution is obtained through a direct application of the Bayes' rule. This recursion requires the specification of a dynamic model (see section 2) describing the state evolution, $p\left(\boldsymbol{x}_{k} \mid \boldsymbol{x}_{k-1}\right)$ and a model that gives the likelihood of any state in the light of the current observation, $p\left(\boldsymbol{z}_{k} \mid \boldsymbol{x}_{k}\right)$. The recursion is initialised with some distribution for the initial state $p\left(\boldsymbol{x}_{0}\right)$. Once the sequence of filtering distribution is known, point estimates of the state can be obtained according to any appropriate function, leading for example to the Maximum a Posteriori (MAP) estimate, $\arg \max _{\boldsymbol{x}_{k}} p\left(\boldsymbol{x}_{k} \mid \boldsymbol{Z}_{k}\right)$, and to the Minimum Mean Square Error (MMSE) estimate, $\int \boldsymbol{x}_{k} p\left(\boldsymbol{x}_{k} \mid \boldsymbol{Z}_{k}\right) d \boldsymbol{x}_{k}$.

The basic idea behind the particle filter is very simple. Starting with a weighted set of samples $\left\{\boldsymbol{x}_{k-1}^{(i)}, w_{k-1}^{(i)}\right\}_{i=1}^{N}$ approximately distributed according to $p\left(\boldsymbol{x}_{k-1} \mid \boldsymbol{Z}_{k-1}\right)$, new samples are generated from a suitable proposal distribution, which may depend on the previous state and the new measurements, i.e., $\boldsymbol{x}_{k}^{(i)} \sim q_{p}\left(\boldsymbol{x}_{k} \mid \boldsymbol{x}_{k-1}^{(i)}, \boldsymbol{z}_{k}\right), i=1, \ldots, N$. To maintain a consistent sample, the new importance weights are set to

$$
w_{k}^{(i)} \propto w_{k-1}^{(i)} \frac{p\left(\boldsymbol{z}_{k} \mid \boldsymbol{x}_{k}^{(i)}\right) p\left(\boldsymbol{x}_{k}^{(i)} \mid \boldsymbol{x}_{k-1}^{(i)}\right)}{q_{p}\left(\boldsymbol{x}_{k} \mid \boldsymbol{x}_{k-1}^{(i)}, \boldsymbol{z}_{k}\right)},
$$

with $\sum_{i=1}^{N} w_{k}^{(i)}=1$. The new particle set $\left\{\boldsymbol{x}_{k}^{(i)}, w_{k}^{(i)}\right\}_{i=1}^{N}$ is then approximately distributed according to $p\left(\boldsymbol{x}_{k} \mid \boldsymbol{Z}_{k}\right)$. The performance of the particle filter depends on the quality of the proposal distribution. In this paper, we use the state evolution model $p\left(\boldsymbol{x}_{k} \mid \boldsymbol{x}_{k-1}\right)$ as a proposal distribution and this makes the new importance weights in (5) become proportional to the corresponding particle likelihoods. This implementation of the sequential Monte Carlo method corresponds to the bootstrap filter as proposed in [19].

\section{Joint Probability Data Association}

Data association is a problem of crucial importance for multiple target tracking because of the necessity to relate each measurement to the correct object. Several methods have been proposed in the estimation and tracking literature [9], [10]. In general multi-target tracking deals with state estimation of an unknown number of targets. Some methods consider special cases with a constant or known number of targets. The observations are assumed to originate from different targets or from clutter. The clutter is a special type of the so-called false alarms, whose statistical properties are different from the targets. In some applications only one measurement is assumed available from each target, where in other applications several returns are available. This will of course reflect which data association method to use.

Several classical data association methods exist [20]. The simplest is the nearest neighbour (NN) method. In [9], this is referred to as the nearest neighbour standard filter and it uses only the closest observation to any given state to perform the measurement update step. The method can also be implemented as a global optimisation, so that the total number of observations for tracking the statistical distance is minimised. Another multi-target tracking association method is the Joint Probabilistic Data Association (JPDA) which is an extension of the Probability Data Association algorithm [9] to multiple targets. It estimates the states by a sum over all the association hypothesis weighted by the probabilities from the likelihood. The most general data association method is a computationally intensive algorithm called the multiple hypothesis tracking (MHT), which calculates every possible update hypothesis [21]. In [22], the so-called probabilistic MHT (PMHT) method is presented, using a maximum-likelihood method in combination with the expectation maximisation (EM) method. A comparative study of JPDAF and PMHT is made in [23].

In [24], the solution to the assignment problem for data association is proposed within the Bayesian framework by incorporating the association in the estimation equations. In [25], [26], this idea is suggested for particle filtering, when the problem of maintaining a track on a target in the presence of intermittent spurious objects. Samples are drawn from the overall target probability density. The so-called joint-filter in [27] is a solution to the joint data association and estimation problem for particle filters. The estimation is performed by a particle filter and a Gibbs sampler [28] is used for the data association. The case with unknown number of targets is handled by a multiple hypotheses test.

In this paper, the JPDA approach is used, which is an extension of the PDA approach [9], [10] for single targets. In the JPDA, a known number of targets $\tau$ is assumed. The index $t=\{1, \ldots, \tau\}$ designates one among the $\tau$ targets. The measurements at time step $k$ are denoted as $\boldsymbol{z}_{k}=\left\{\boldsymbol{z}_{k}^{j}\right\}_{j=0}^{m_{k}}$, where an artificial measurement $\boldsymbol{z}_{0}$ is introduced to handle false alarms or clutter and the number of measurements is given by $m_{k}$. The measurement to target association probabilities are evaluated jointly across the targets. Let $\theta$ denotes the joint association event (the time index $k$ is omitted for simplicity) and $\theta_{t}^{j}$ is the particular event which assigns the measurement $j$ to the target $t$. By assuming that the estimation problem is Markovian and by applying the Bayes' theorem, the joint association probabilities are

$$
P\left(\theta \mid \boldsymbol{Z}_{k}\right)=\frac{1}{c} p\left(\boldsymbol{z}_{k} \mid \theta, \boldsymbol{X}_{k}\right) P\left(\theta \mid \boldsymbol{X}_{k}\right),
$$

where $c$ is a normalisation constant and $\boldsymbol{X}_{k}=\left\{\boldsymbol{x}_{1}, \ldots, \boldsymbol{x}_{k}\right\}$. Additionally, it is assumed that the measurements are detected independently of each other. The probability of the assignment $\theta$ conditioned on the sequence of the targets' states $P\left(\theta \mid \boldsymbol{X}_{k}\right)$ is approximated by

$$
P\left(\theta \mid \boldsymbol{X}_{k}\right)=P_{D}^{\tau-n}\left(1-P_{D}\right)^{n} P_{F A}^{m_{k}-(\tau-n)},
$$


where $P_{D}$ denotes the probability of detection, $n$ is the number of $z_{0}$ assignments and $P_{F A}$ denotes the probability of false alarm.

The innovation between the $j$-th measurement $z_{k}^{j}$ and the predicted measurement $\hat{z}_{t, k}^{j}$ of target $t$ at time step $k$ is $\boldsymbol{\nu}_{t, k}^{j}=\boldsymbol{z}_{k}^{j}-\hat{\boldsymbol{z}}_{t, k}^{j}$ and $\boldsymbol{S}_{t, k}^{j}$ is the corresponding innovation covariance matrix. Then the normalised innovation $d_{t, k}^{j}$ is defined as

$$
d_{t, k}^{j}=\left(\boldsymbol{\nu}_{t, k}^{j}\right)^{T}\left(\boldsymbol{S}_{t, k}^{j}\right)^{-1} \boldsymbol{\nu}_{t, k}^{j} .
$$

By assuming that the measurement is of dimension $M$, it follows that the $M$-dimensional Gaussian association likelihood for the $j$-th measurement to the target $t$

$$
p\left(\boldsymbol{\nu}_{t, k}^{j}\right)=\frac{1}{(2 \pi)^{M / 2}\left|\boldsymbol{S}_{t, k}^{j}\right|^{1 / 2}} \exp \left\{\frac{-\left(d_{t, k}^{j}\right)^{2}}{2}\right\},
$$

where $\left|\boldsymbol{S}_{t, k}^{j}\right|$ is the determinant of $\boldsymbol{S}_{t, k}^{j}$.

Finally, the probability of an individual joint association event is given by

$$
P\left(\theta \mid \boldsymbol{Z}_{k}\right)=P_{D}^{\tau-n}\left(1-P_{D}\right)^{n} P_{F A}^{m_{k}-(\tau-n)} \prod_{\theta_{t}^{j} \in \theta} p\left(\boldsymbol{\nu}_{t, k}^{j}\right) .
$$

As shown in [29], the number of associations increases exponentially with an increase in the number of measurements and targets, rendering the use of all hypothesis infeasible for even moderate values for these quantities. The number of possible associations $\lambda$, given that $N_{D}$ of the $\tau$ targets have been detected, is

$$
N_{\lambda_{k}}\left(N_{D}, \tau\right)=\frac{m_{k} ! \tau !}{N_{D} !\left(m_{k}-N_{D}\right) !\left(\tau-N_{D}\right) !}
$$

and so the total number of possible hypotheses (since $N_{D}$ is not known) is

$$
\sum_{N_{D}=0}^{\min \left(m_{k}, \tau\right)} N_{\lambda_{k}}\left(N_{D}, \tau\right)
$$

Even for the case of three targets and three measurements, this amounts to 34 hypotheses. Therefore, in practice, it is common to apply gating techniques [9] to reduce the number of hypotheses and the computational cost respectively.

\section{Monte Carlo JPDA}

In this subsection, we describe the Monte Carlo JPDA used in our tracking algorithm. The Monte Carlo version of JPDA has been studied by several authors (see e.g. [30], [31]). A recent paper of Vermaak et al. [32] surveys most of the approaches developed earlier. In [32] marginal filtering distributions for each of the targets are represented with Monte Carlo samples, or particles, instead of based on a Gaussian approximation, as it is done in the standard JPDAF. The JPDA particle filter implemented in our paper is given below:

\section{The JPDA particle filter for multiple target tracking}

1. Initialisation.

Set $k=0$, generate $N$ samples $\boldsymbol{x}_{t, 0}^{(i)}$ for all targets $t=1, \ldots, \tau$ independently. $\boldsymbol{x}_{t, 0}^{(i)}$ is drawn from $p\left(\boldsymbol{x}_{t, 0}\right)$, for $i=1, \ldots, N$ particles

2. For $t=1, \ldots, \tau$ targets,

For $j=0, \ldots, m_{k}$ measurements

Compute $\beta_{t, k}^{j}=\sum_{\theta_{t, k}^{j} \in \theta} P\left(\theta \mid Z_{k}\right)$ as the summation over all the joint events in which the marginal event $\theta_{t, k}^{j}$ of interest occurs.

For $i=1, \ldots N$ particles compute the independent weights for each particle according to $w_{t, k}^{(i)}=\sum_{j=0}^{m_{k}} \beta_{t, k}^{j} p\left(\boldsymbol{z}_{k}^{j} \mid \boldsymbol{x}_{t, 0}^{(i)}\right)$ and normalise the weights for each target: $\tilde{w}_{t, k}^{(i)}=\frac{w_{t, k}^{(i)}}{\sum_{i=1}^{N} w_{t, k}^{(i)}}$.

For each target, generate a new set $\left\{\boldsymbol{x}_{t, k}^{(i *)}\right\}_{i=1}^{N}$, by resampling with $N$ times from $\left\{\boldsymbol{x}_{t, k}^{(i)}\right\}_{i=1}^{N}$, where $P\left(\boldsymbol{x}_{t, k}^{(i *)}=\boldsymbol{x}_{t, k}^{(i), t}\right)=\tilde{w}_{t, k}^{(i)}$.

For $i=1, \ldots N$ predict new particles

$$
\boldsymbol{x}_{t, k+1}^{(i)}=\boldsymbol{F} \boldsymbol{x}_{t, k}^{(i *)}+\boldsymbol{v}_{t, k}^{(i)} .
$$

End For

End For

3. Increase $k$ and iterate to step 2 .

The algorithm assumes knowledge of the maximum number of targets, $\tau$. It starts with a single filter with uniformly distributed particles across the image scene. The variance of this filter is used to monitor the convergence of the filter. Once this filter shows convergence (by convergence we mean that the variance of the $\mathrm{PF}$, is less than a certain threshold value) a new filter is initialised with particles a priori uniformly distributed across the image except at the region around the target tracked by the first filter. The prior around the region tracked by the first filter (the exclusion region) is zero. This selection of prior distribution avoids the likelihood of two filters tracking the same object. The variance of this second filter monitors the convergence of the new filter. Once the second filter reaches convergence, another filter is initialised with a prior from the region not covered by the first two filters. During the tracking process the number of objects and 
the identity of active objects are estimated as shown in the next paragraph. This is used to detect when an object disappears from the scene and to stop the corresponding tracking filter.

Let $P\left(H_{t} \mid \boldsymbol{Z}_{k}\right)(t=1, \ldots, \tau)$ denote the posterior probability of the existence of $t$ number of targets. In general, this probability depends on the 'full' hypothesis list which considers all possible hypotheses from frame 1 to frame $k$. However, it can be approximately estimated from the hypotheses assumed in the Monte Carlo JPDA. According to the total probability theorem, the existence probability of $t$ number of targets is given by

$$
P\left(H_{t} \mid \boldsymbol{Z}_{k}\right)=\sum_{\theta \in \chi_{t}} P\left(\theta \mid \boldsymbol{Z}_{k}\right), t=1, \ldots, \tau .
$$

where $\chi_{t}$ is the event (hypothesis) that $t$ number of targets exists. Similarly to this, joint existence probabilities of specific targets (such as the existence of target 1 with target 2 or target 1 with target 3 ) also can be estimated. Let $H_{t_{1}, t_{2}, \ldots, t_{\rho}}$ be the hypothesis that $t_{1}, t_{2}, \ldots, t_{\rho}$ targets exist, with $\rho$ being the number of active targets. Then the existence probability of this event is given by:

$$
P\left(H_{t_{1}, t_{2}, \ldots, t_{\rho}} \mid \boldsymbol{Z}_{k}\right)=\sum_{\theta \in \chi_{t_{1}, t_{2}, \ldots, t_{\rho}}} P\left(\theta \mid \boldsymbol{Z}_{k}\right),
$$

where $\chi_{t_{1}, t_{2}, \ldots, t_{\rho}}$ denotes the events that targets $\left\{t_{1}, t_{2}, \ldots, t_{\rho}\right\}$ exist. These probabilities are used for the purpose of track management. If a target (tracked by the algorithm) disappears from the scene, the changes in the number of objects is reflected in the probabilities $P\left(H_{t} \mid \boldsymbol{Z}_{k}\right)$. From the probabilities $P\left(H_{t_{1}, t_{2}, \ldots, t_{\rho}} \mid \boldsymbol{Z}_{k}\right)$, we can obtain the identity of the target which just disappeared. Accordingly, we can stop the corresponding filter and reinitialise for tracking a new target. The use of these probabilities in track management is further explained in the next section with examples.

\section{EXPERIMENTAL RESULTS}

The proposed algorithm is tested over real-world video sequences with a changing number of football players (the same as in [2]). The aim is to track all red players. The maximum number of targets is three $(\tau=3)$. The algorithm is implemented with $N=500$ samples for each filter, with $U=128 \times 128 \times 128$ number of bins for the colour cue, with a system noise covariance $Q=\operatorname{diag}\{15,15\}$ and a measurement noise covariance $\sigma^{2}=0.1^{2}$. The number of measurements available is $m_{k}=3$ and they are obtained from image regions specified by the state vector. When the number of objects being tracked is less than $m_{k}=3$, the remaining observations are image regions pointing to a region with no relevant colour information. This serves as clutter in the visual tracking process.

Figure 3 shows several frames to illustrate the work of the proposed algorithm. At frame 1, one filter is initialised with random samples uniformly spread all over the image frame.
As shown in Figure 1 (frame 20), this filter initially attempts to track all red players but gradually converges to the red person on the right (frame 40). At frame 41, a new filter is initialised with an initial prior distribution uniformly distributed across all regions except the region around the already tracked object. The frame 41 shows these samples. This selection of the initial prior avoids the occurrence of a new filter tracking the already tracked person by the first filter. At frame 52, both filters are tracking two persons and at frame 53, a new filter (third filter) is initialised with a prior covering all regions except two regions already tracked by first two filters. After ten frames (at frame 63), all three objects are tracked by the proposed algorithm.

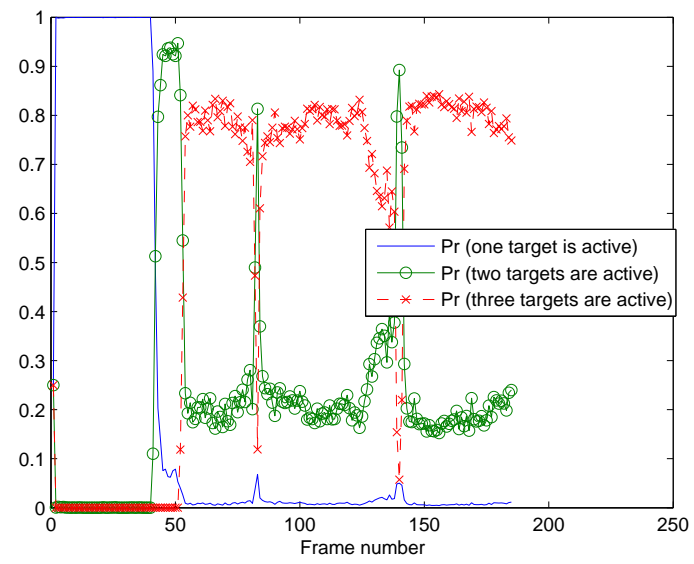

Figure 1. Probabilities of the number of targets: one active object; two active targets or three active targets

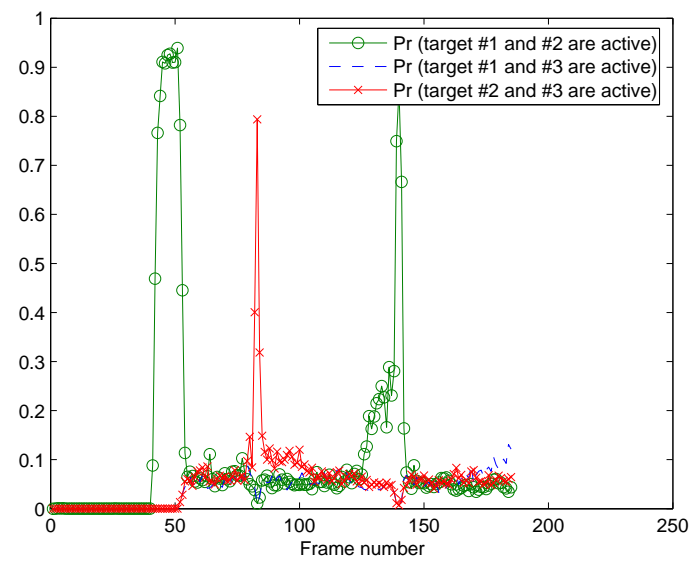

Figure 2. Probabilities of joint existence of possible two targets

At frame 82, the person tracked by the first filter leaves the scene. This is also indicated by the changes in the joint existence probabilities shown in Figure 1. As seen from the Figure 1 , the dominant probability changes from the probability of having three objects to the the probability of having two objects. As seen from frame 83, the first filter was stopped when the person disappeared. The same filter is initialised to 

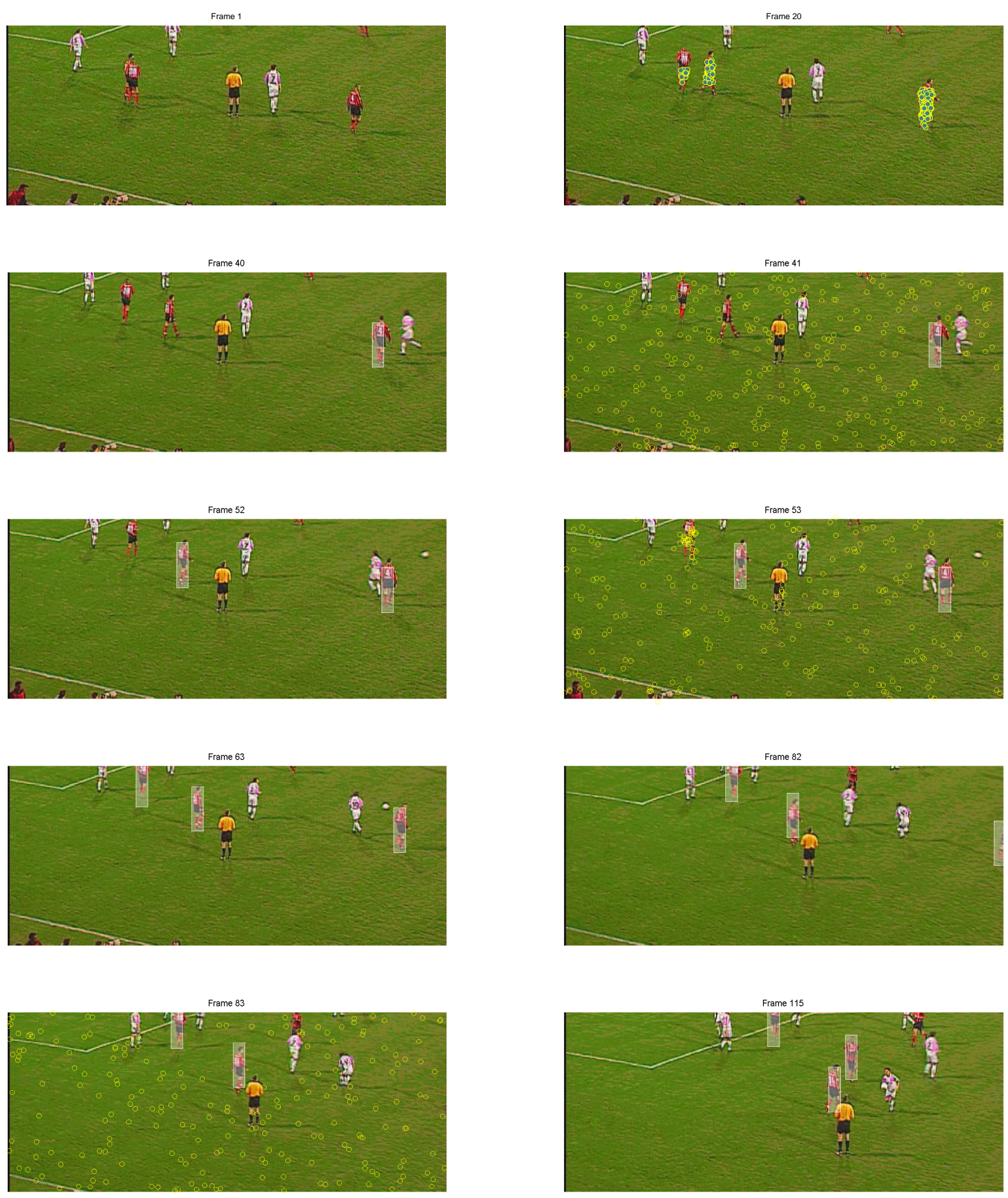

Figure 3. Image frames of a football sequence: detected and tracked players are marked with a rectangle and the circles denote the locations of particles 
track any new objects appearing in the image scene. The initialisation is similar to the earlier one and the initial samples are obtained from regions not covered by the two tracking filters (the 2nd and the 3rd). It is important to know which object disappeared and which filter is reinitialised. Figure 2 shows different existence probabilities of having two objects (i.e., probabilities of having object 1 with 2 , 1 with 3 , and 2 with 3). Figure 2 indicates also that around the frame 82 the probability of having objects 2 and 3 is very high compared to other possibilities. This illustrates that the object 1 is the disappeared object and we can reinitialise then filter 1.

\section{Discussion}

Advantages of the proposed algorithm are its computational simplicity. The technique developed can be extended to deal with full occlusions, e.g. when the targets cross their paths. In order to be able to cope with full occlusions, unique features from each target are required, which is an open issue for future research. Additionally, separate detection techniques, such as image segmentation, can be used to detect the targets and to speed up the process of target track initiation. Investigations are currently going on the development of adaptive cues able to deal with changeable illumination on the scene.

\section{CONCLUSIONS}

This paper presents a JPDA particle filter for multiple targets tracking in video sequences. The experimental results from real video sequences show its reliable performance. The algorithm is characterised with low computational complexity and is able to cope with partial occlusions and recover after temporary loss. We evaluate the number of objects in the current frame and the track management is handled using probabilities of the number of objects in the same frame.

Acknowledgements. The authors are grateful to the financial support by the UK MOD Data and Information Fusion Defence Technology Centre.

\section{REFERENCES}

[1] P. Brasnett, L. Mihaylova, N. Canagarajah, and D. Bull, "Particle filtering for multiple cues for object tracking in video sequences," in Proc. of the 17th SPIE Annual Symposium on Electronic Imaging, Science and Technology, 2005, vol. 5685, pp. 430-440.

[2] J. Czyz, B. Ristic, and B. Macq, "A color-based particle filter for joint detection and tracking of multiple objects," in Proc. of the ICASSP, 2005.

[3] E. Stringa and C.S. Regazzoni, "Real-time video-shot detection for scene surveillance applications," IEEE Trans. Image Processing, vol. 9, pp. 69-79, Jan. 2000.

[4] I. Haritaoglu, D. Harwood, and L. Davis, " $w^{4}$ : who? when? where? what? a real time system for detecting and tracking people," in Proc. IEEE Conf. Face Gesture Recognition, 1998, pp. 222-227.
[5] D. Koller, J. Weber, T. Huang, J. Malik, B. Rao G. Ogasawara, and S. Russell, "Toward robust automatic traffic scene analysis in real-time," in Proc. Int. Conf. Pattern Recog., 1994, vol. 1, pp. 126-131.

[6] J. Vermaak, S. Maskell, M. Briers, and P. Pérez, “A unifying framework for multi-target tracking and existence," in Proc. of the 8th International Conf. on Information Fusion, 2005.

[7] M. Isard and A. Blake, "A mixed-state condensation tracker with autmatic model-switching," in Proc. Int. Conf. Computer Vision, 1998, pp. 107-112.

[8] M. Isard and J. MacCormick, "Bramble: a Bayesian multiple blob tracker," in Proc. Int. Conf. Computer Vision, 2001, pp. 34-41.

[9] Y. Bar-Shalom and X.R. Li, Estimation and Tracking: Principles, Techniques and Software, Artech House, Boston, MA, 1993.

[10] S. Blackman and R. Popoli, Design and Analysis of Modern Tracking Systems, Artech House, 1999.

[11] W. Ng, J. Li, S. Godsill, and J. Vermaak, "A review of recent results in multiple target tracking," in Proc. of the International Symp. on Signal Processing and Analysis, 2005.

[12] W. Ng, J. Li, S. Godsill, and J. Vermaak, “Tracking variable number of targets using sequential monte carlo methods," in Proc. of IEEE Statistical Signal Processing Workshop, 2005.

[13] Bar-Shalom and W. Blair, Eds., Multitarget-Multisensor Tracking: Allpications and Advances, Artech House, 2000.

[14] D. Comaniciu, V. Ramesh, and P. Meer, "Real-time tracking of non-rigid objects using mean shift," in Proc. IEEE Conf. Comp. Vis. Pattern Rec., 2000, pp. 142-149.

[15] K. Nummiaro, E. Koller-Meir, and L. Van-Gool, "An adaptive color-based particle filter," Image and Vision Computing, vol. 21, pp. 99-110, 2003.

[16] M. Isard and A. Blake, "Contour tracking by stochastic propagation of conditional density," in European Conf. on Comp. Vis. 1996, pp. 343-356, Cambridge, UK.

[17] M. Isard and A. Blake, "Condensation - conditional density propagation for visual tracking," Intl. Journal of Computer Vision, vol. 28, no. 1, pp. 5-28, 1998.

[18] P. Pérez, J. Vermaak, and A. Blake, "Data fusion for tracking with particles," Proceedings of the IEEE, vol. 92, no. 3, pp. 495-513, March 2004.

[19] N. Gordon, D. Salmond, and A. Smith, "Novel approach to nonlinear/ non-Gaussian Bayesian state estimation," IEE Proc. $-F$, vol. 140, no. 2, pp. 107-113, 1993.

[20] T. Kirubarajan and Y. Bar-Shalom, "Probabilistic data association techniques for target tracking in clutter," Proc. of the IEEE, vol. 92, no. 3, pp. 536-557, 2004. 
[21] D. Reid, "An algorithm for tracking multiple targets," IEEE Trans. Aut. Contr., vol. 24, no. 6, pp. 84-90, 1979.

[22] H. Gauvrit, J-P. Le Cadre, and C. Jauffret, "A formulation of multitarget tracking as an incomplete data problem," IEEE Trans. Aerospace and Systems, vol. 33, no. 4, pp. 1242-1257, Oct. 1997.

[23] C. Rago, P. Willett, and R. Streit, "A comparison of the JPDAF and PMHT tracking algorithms," in Proc. of the ICASSP, 1995, vol. 5, pp. 3571-3574.

[24] D. Avitzour, "A stochastic simulation bayesian approach to multitarget tracking," IEE Proc. Radar, Sonar and Navig., vol. 142, pp. 41-44, 1995.

[25] D.J. Salmond, D. Fisher, and N.J. Gordon, "Tracking om the presence of intermittant spurious objects and clutter," in SPIE Conf. on Signal and Data Processing of Small Targets, 1998.

[26] N.J. Gordon, "A Hybrid Bootstrap Filter for Target Tracking in Clutter," IEEE Trans. on Aerospace and Electronic Systems, vol. 33, no. 1, pp. 353-358, 1997.

[27] C. Hue, J-P. Le Cadre, and P. Perez, "Tracking multiple objects with particle filtering," IEEE Trans. Aerospace and Systems, vol. 38, no. 3, pp. 791-812, July 2002.

[28] S. Gemen and D. Gemen, "Stochastic relaxation, Gibbs distributions and the Bayesian restoration of images," IEEE Transactions on Pattern Analysis and Machine Intelligence, vol. 6, pp. 721-741, 1984.

[29] B.Ristic, S. Arulampalam, and N.Gordon, Beyond the Kalman Filter: Particle Filters for Tracking Applications, Artech House Radar Library, 2004.

[30] R. Karlsson and F. Gustafsson, "Monte Carlo data association for multiple target tracking," in IEE Target Tracking: Algorithms and Applications, Netherlands, Oct 2001.

[31] D. Schultz, W. Burgard, D. Fox, and A.B. Cremers, "Tracking multiple moving targets with a mobile robot using particle filters and statistical data association," in IEEE Internation. Conf. on Robotics and Autom., 2001.

[32] J. Vermaak, S.J. Godsill, and P. Perez, "Monte Carlo filtering for multi-target tracking and data assoication," IEEE Trans. Aerospace and Systems, vol. 41, no. 1, pp. 309-332, January 2005.

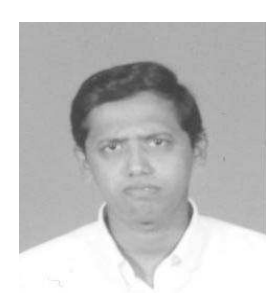

Mohamed Jaward received the PhD degree in Automatic Control and Systems Engineering from the University of Sheffield, UK in 2002. He is a Research Assistant at the Department of Electrical and Electronic Engineering, University of Bristol, UK. His research interests include multiple models, sequential Monte Carlo methods, target tracking in video sequences, fault detection and data detection in communication systems.

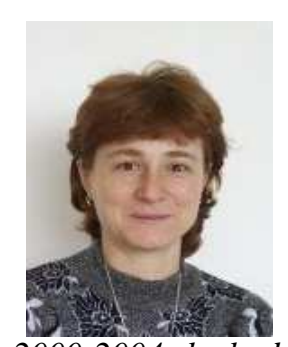

Lyudmila Mihaylova obtained her MSc and PhD degree in Systems and Control Engineering from the Technical University of Sofia, Bulgaria. From 19962000 she was a Research Scientist at the Bulgarian Academy of Sciences and as a part-time Assistant Professor at Technical University of Sofia. In the period 2000-2004 she had temporary positions as a Research Associate at Katholieke Universiteit Leuven and at the University of Ghent, Belgium. Since February 2004 she has been a Research Fellow at Bristol University, UK. Her interests are in the area of nonlinear filtering, statistical signal processing, sensor data fusion, machine learning, control and intelligent autonomous systems. She has authored over 50 papers in conference proceedings and journals. Dr. Mihaylova is a member of the IEEE, Signal Processing Society and of the International Society of Information Fusion.

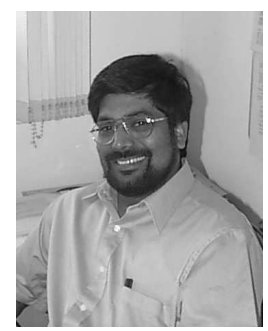

Nishan Canagarajah is currently a Professor of Multimedia Signal Processing at Bristol. Prior to this he was an RA and lecturer at Bristol investigating DSP aspects of mobile radio receivers. He has BA (Hons) and a PhD in DSP Techniques for Speech Enhancement both from the University of Cambridge. His research interests include image and video coding, image segmentation, content based video retrieval, $3 D$ video, image fusion and the application of signal processing to audio and medical electronics. He is widely supported in these areas by industry, EU and the EPSRC. He has been involved in a number of FP5 and FP6 projects where the team has been developing novel image/video processing algorithms. He has published more than 150 papers and two books. He is a member of the EPSRC College.

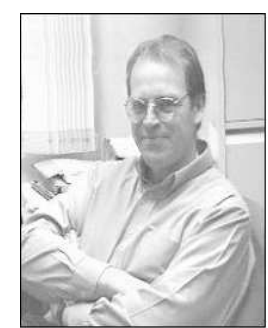

David Bull is Professor of Signal Processing and Head of the Electrical and Electronic Engineering Department at the University of Bristol. Prior to his current appointment he has been a Systems Engineer at Rolls Royce and subsequently a Lecturer at Cardiff University. He leads the Signal Processing activities within the Centre for Communications Research where he is Deputy Director. He has been a member of the UK Foresight Panel and the Steering Group for the DTI/EPSRC LINK programme in Broadcast Technology and is currently on the EPSRC Peer Review College. He is a past director of the VCE in Digital Broadcasting and Multimedia Technology and is currently Chairman and Technical Director of ProVision Communication Technologies Ltd., specialising in wireless multimedia communications. David Bull has worked widely in the fields of 1 and 2-D signal processing and has published over 250 papers, various articles and 2 books. He has won two IEE Premium awards for this work. His current research is focused on the problems of image and video communications for low bit rate wireless, internet and broadcast applications. 